\title{
A new method for complete quantitative interpretation of gravity data due to dipping faults
}

\author{
El-Sayed ABDELRAHMAN, Mohamed GOBASHY, Eid ABO-EZZ, \\ Tarek EL-ARABY \\ Geophysics Department, Faculty of Science, Cairo University, Egypt; \\ e-mail: sayed5005@yahoo.com, bouguer3000@yahoo.com, aboezz2000@yahoo.com, \\ tmelaraby@sci.cu.edu.eg
}

\begin{abstract}
We have developed a simple method to determine completely the model parameters of a buried dipping fault from gravity data (depths to the centers of the upper and lower portions of the faulted thin slab, dip angle, and amplitude coefficient). The method is based on defining the anomaly values at the origin and at four symmetrical points around the origin on the gravity anomaly profile. By defining these five pieces of information, the dip angle is determined for each value of the depth of the lower portion of the faulted thin slab by solving iteratively one nonlinear equation of the form $f(\alpha)=0$. The computed dip angles are plotted against the values of the depth representing a continuous depth-dip curve. The solution for the depth to the lower portion of the faulted thin slab (down-thrown block) and the dip angle of the buried fault is read at the common intersection of the depth-dip curves. Knowing the depth to the center of the lower portion of the faulted layer and the dip angle, the problem of determining the depth to the center of the upper portion of the faulted slab (up-thrown block) is transformed into the problem of solving iteratively a nonlinear least-squares equation, $f(z)=0$. Because the depths and the dip angle are known, the amplitude coefficient, which depends on the thickness and density contrast of the thin slab, is determined using a linear least-squares equation. The method is applied to theoretical data with and without random errors. The validity of the method is tested on real gravity data from Egypt. In all cases examined, the model parameters obtained are in good agreement with the actual ones and with those given in the published literature.
\end{abstract}

Key words: gravity interpretation, dipping faults, iterative methods, least squares method, depth-curves method, noise

\section{Introduction}

The dipping fault model is frequently used in gravity interpretation to find the depth and the dip angle of a class of faulted structures. Estimation 
of these parameters from gravity anomalies has drawn considerable attention. Fault interpretation using gravity modeling methods, e.g., 2D (Tanner, 1967), 2.5D (Chakravarthi, 2011), or 3D (Cordell and Henderson, 1968) involves personal judgments and requires density information as part of the input, along with depth and dip angle information obtained from geological and/or other geophysical data. Other methods use fixed simple geometry for interpreting gravity anomalies due to dipping faults (Geldart et al., 1966; Paul et al., 1966; Green, 1976; Thompson, 1982; Gupta, 1983; Lines and Treitel, 1984; Abdelrahman et al., 1989; Gupta and Pokhriyal, 1990; Reid et al., 1990; Abdelrahman et al., 2003; Phillips et al., 200\%; Utyupin and Mishenin, 2012). The advantage of fixed geometry methods over continuous modeling methods is that they require neither density, dip angle, nor depth information, and they can be applied if little or no factual information other than the gravity data is available.

Very recently, Abdelrahman et al. (2013), Essa (2013) and Abdelrahman and Essa (2015) developed methods to determine only the depth to the upper portion of the faulted thin slab as well as the dip angle of the faulted structure from gravity data using numerical techniques. However, the drawback of these methods is that they assume that the throw of the fault is extremely large, i.e., the depth to the lower portion of the thin slab approaches infinity. This assumption will lead definitely to large errors in estimating the model parameters of the faulted structure from real gravity data.

In this paper, we have developed a simple method to determine completely the model parameters of a buried dipping fault from the residual gravity anomaly. Using the anomaly values at the origin and four symmetrical points on the gravity anomaly profile, the problem of determining the depth to the center of the lower portion of the thin slab and the dip angle is transformed into the problem of solving one nonlinear equation to construct depth-dip angle curves. Knowing the depth and the dip angle computed from the constructed curves, the problem of determining the depth to the center of the upper portion of the faulted thin slab determination is transformed into the problem of solving iteratively a nonlinear least-squares equation, whereas the amplitude coefficient is determined using a simple linear least-squares equation. The accuracy of the result abstained by the procedures depends upon the accuracy to which the origin of the residual 
anomaly profile can be determined from geological and/or other geophysical data. Also for this method to be valid, we assume that the thickness of the faulted layer is small compared to the depth of the up-thrown faulted block, i.e. the layer can be approximated by a thin sheet located at its center. The thin sheet approximation is valid and correct within $2 \%$ if the thickness is equal to or less than the depth. The method is applied to theoretical data with and without random errors. The validity of the method is tested on real gravity data from Egypt.

\section{The method}

The formula for the residual gravity anomaly generated along the profile normal to the strike of a 2-D dipping faulted thin slab having infinite strike length is given by the following equation (Telford et al., 1976):

$g\left(x_{i}, z, h, \alpha\right)=K\left[\pi+\operatorname{atan}\left(\frac{x_{i}}{z}+\cot \alpha\right)-\operatorname{atan}\left(\frac{x_{i}}{h}+\cot \alpha\right)\right]$,

where $z$ and $h$ are the depths to the centers of the upper and the lower portions of the layer, respectively, $\alpha$ is the angle of dip measured counterclockwise from surface, $K=2 \gamma \sigma t$, is the amplitude coefficient related to the thickness $(t)$ and density contrast $(\sigma)$ of the faulted slab, $\gamma$ is the universal gravitational constant, and $x_{i}$ is the horizontal coordinate position. The two-dimensional gravity dipping fault model is shown in Fig. 1.

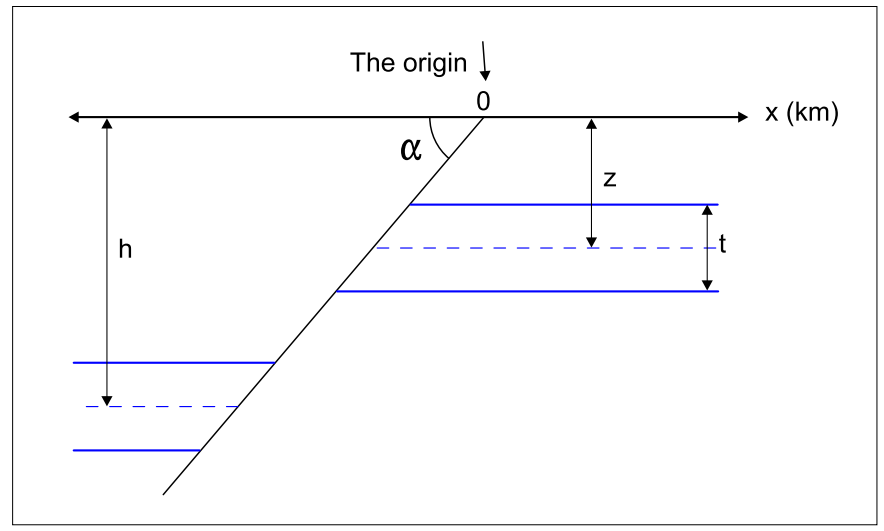

Fig. 1. Geometry of the dipping faulted thin slab model. 
Equation (1) can be written as:

$g\left(x_{i}, z, h, \alpha\right)=\frac{g(0)}{\pi}\left[\pi+\operatorname{atan}\left(\frac{x_{i}}{z}+\cot \alpha\right)-\operatorname{atan}\left(\frac{x_{i}}{h}+\cot \alpha\right)\right]$,

where $g(0)=K \pi$ is the anomaly value at the origin of the residual anomaly profile.

Equation (2) gives the following values at $x_{i}= \pm N$ and $\pm M$ :

$g(N)=\frac{g(0)}{\pi}\left[\pi+\operatorname{atan}\left(\frac{N}{z}+\cot \alpha\right)-\operatorname{atan}\left(\frac{N}{h}+\cot \alpha\right)\right]$,

$g(-N)=\frac{g(0)}{\pi}\left[\pi+\operatorname{atan}\left(\frac{-N}{z}+\cot \alpha\right)-\operatorname{atan}\left(\frac{-N}{h}+\cot \alpha\right)\right]$,

$g(M)=\frac{g(0)}{\pi}\left[\pi+\operatorname{atan}\left(\frac{M}{z}+\cot \alpha\right)-\operatorname{atan}\left(\frac{M}{h}+\cot \alpha\right)\right]$,

$g(-M)=\frac{g(0)}{\pi}\left[\pi+\operatorname{atan}\left(\frac{-M}{z}+\cot \alpha\right)-\operatorname{atan}\left(\frac{-M}{h}+\cot \alpha\right)\right]$,

where $g(N), g(-N), g(M)$, and $g(-M)$ are the anomaly values at four symmetrical points around the origin.

Equations (3-6), can be simplified as:

$\tan \left[\pi D(N)+\operatorname{atan}\left(\frac{N}{h}+\cot \alpha\right)\right]-\cot \alpha=\frac{N}{z}$,

$\tan \left[\pi D(-N)+\operatorname{atan}\left(\frac{-N}{h}+\cot \alpha\right)\right]-\cot \alpha=\frac{-N}{z}$,

$\tan \left[\pi D(M)+\operatorname{atan}\left(\frac{M}{h}+\cot \alpha\right)\right]-\cot \alpha=\frac{M}{z}$,

$\tan \left[\pi D(-M)+\operatorname{atan}\left(\frac{-M}{h}+\cot \alpha\right)\right]-\cot \alpha=\frac{-M}{z}$,

where

$D(N)=\frac{g(N)}{g(0)}-1, \quad D(-N)=\frac{g(-N)}{g(0)}-1$, 
$D(M)=\frac{g(M)}{g(0)}-1, \quad D(-M)=\frac{g(-M)}{g(0)}-1$.

Adding Eq. (7) to Eq. (8), and adding Eq. (9) to Eq. (10) will eliminate (z) and the following equations are obtained:

$$
\begin{aligned}
\tan [\pi D(N) & \left.+\operatorname{atan}\left(\frac{N}{h}+\cot \alpha\right)\right]+ \\
& +\tan \left[\pi D(-N)+\operatorname{atan}\left(\frac{-N}{h}+\cot \alpha\right)\right]=2 \cot \alpha
\end{aligned}
$$

and

$$
\begin{aligned}
\tan [\pi D(M) & \left.+\operatorname{atan}\left(\frac{M}{h}+\cot \alpha\right)\right]+ \\
& +\tan \left[\pi D(-M)+\operatorname{atan}\left(\frac{-M}{h}+\cot \alpha\right)\right]=2 \cot \alpha .
\end{aligned}
$$

Using equations (11-12), we obtain after simple mathematical manipulation the following nonlinear equation in the dip angle $(\alpha)$ and the depth to the center of the lower portion of the faulted thin slab $(h)$.

$\alpha=\operatorname{datan}\left(\tan \left\langle\operatorname{atan}\left(T_{1}+T_{2}-T_{3}\right)-\pi D(-M)\right\rangle+\frac{M}{h}\right)$,

where

$$
\begin{aligned}
& T_{1}=\tan \left[\pi D(N)+\operatorname{atan}\left(\frac{N}{h}+\cot \alpha\right)\right], \\
& T_{2}=\tan \left[\pi D(-N)+\operatorname{atan}\left(\frac{-N}{h}+\cot \alpha\right)\right], \\
& T_{3}=\tan \left[\pi D(M)+\operatorname{atan}\left(\frac{M}{h}+\cot \alpha\right)\right] .
\end{aligned}
$$

The dip angle $(\alpha)$ can be obtained by solving Eq. (13) using a simple iteration method (Press et al., 200\%) if $h$ is known. The iterative form of equation (13) is given as:

$\alpha_{f}=f(\alpha j)$

where $\left(\alpha_{j}\right)$ is the initial dip and $\left(\alpha_{f}\right)$ is the revised dip; $\left(\alpha_{f}\right)$ will be used 
as the $\alpha_{j}$ for the next iteration. The iteration stops when $\left|\alpha_{f}-\alpha_{j}\right| \leq e$, where $e$ is a small predetermined real number close to zero.

Thus, the dip angle of the faulted thin slab is determined by solving one non linear equation $f(\alpha)=0$. Any initial guess for $\alpha$ works well because there is always one minimum, provided that $(h)$ remains fixed in the process.

However, Eq. (13) can be used also not only to determine the dip angle $(\alpha)$ of the buried structure but also to simultaneously estimate the depth $(h)$ to the center of the lower block of the faulted structure. The procedure is as follows:

1. Determine the origin of the observed gravity anomaly profile $\left(x_{i}=0\right)$ using geological and/or other geophysical data.

2. Digitize the observed gravity anomaly profile at several points with a suitable interval.

3. Eq. (13) is then applied to the input data yielding dip solutions $\alpha$ for all possible $h$ values for fixed $N$ and $M$ values. The computed $\alpha$ values are plotted against $h$ values representing a continuous depth-dip curve. The depth-dip curves should intersect at a single point, i.e., the value of $\alpha$ at the point of intersection is the dip angle of the faulted structure, and the value of $h$ gives the depth to the center of the lower portion of the faulted thin slab. Theoretically, any two curves associated with two different values of $N$ and $M$ are just enough to simultaneously determine $\alpha$ and $h$. In practice, more than two values of $N$ and $M$ might be necessary because of the presence of noise in data.

Substituting the computed depth $\left(h_{c}\right)$ and the computed dip angle $\left(\alpha_{c}\right)$ as fixed parameters in Eq. (2), we obtain:

$g\left(x_{i}, z\right)=\frac{g(0)}{\pi} W\left(x_{i}, z\right)$,

where $W\left(x_{i}, z\right)=\left[\pi+\operatorname{atan}\left(\frac{x_{i}}{z}+\cot \alpha_{c}\right)-\operatorname{atan}\left(\frac{x_{i}}{h_{c}}+\cot \alpha_{c}\right)\right]$.

Applying the least-squares method, the unknown $z$ in Eq. (15) can be obtained by minimizing:

$\varphi(z)=\sum_{i=1}^{P}\left[L\left(x_{i}\right)-\frac{g(0)}{\pi} W\left(x_{i}, z\right)\right]^{2}$,

where $L\left(x_{i}\right)$ denoted the observed residual gravity anomaly at $x_{i}$. 
Setting the derivative of $\phi(z)$ to zero with respect to $z$ leads to:

$f(z)=\sum_{i=1}^{P}\left[L\left(x_{i}\right)-\frac{g(0)}{\pi} W\left(x_{i}, z\right)\right] W^{*}\left(x_{i}, z\right)=0, \quad P=1,2,3 \ldots$,

where $W^{*}\left(x_{i}, z\right)=\frac{\mathrm{d}}{\mathrm{d} z} W\left(x_{i}, z\right)$.

Eq. (17) can be solved for $z$ using the standard methods for solving nonlinear equations. Here Eq. (17) is solved by a simple iteration method (Press et al., 200\%). The iterative form of Eq. (17) is given as:

$$
z_{f}=\left(\frac{\sum_{i=1}^{P}\left(\frac{x_{i} L\left(x_{i}\right)}{1+\left(\frac{x_{i}}{z_{j}}+\cot \alpha_{c}\right)^{2}}\right)}{\sum_{i=1}^{P} \frac{g(0)}{\pi}\left[\frac{x_{i}\left(\pi+\operatorname{atan}\left(\frac{x_{i}}{z_{j}}+\cot \alpha_{c}\right)-\operatorname{atan}\left(\frac{x_{i}}{h_{c}}+\cot \alpha_{c}\right)\right.}{z_{j}^{2}\left[1+\left(\frac{x_{i}}{z_{j}}+\cot \alpha_{c}\right)^{2}\right]}\right]}\right)^{1 / 2},
$$

where $z_{j}$ is the initial depth parameter and $z_{f}$ is the revised depth parameter; $z_{f}$ will be use as the $z_{j}$ for the next iteration. The iteration stops when $\left|z_{f}-z_{j}\right| \leq e$, where $e$ is a small predetermined real number close to zero.

Thus, the depth to the center of the upper portion of the faulted thin slab is determined by solving one non linear equation $f(z)=0$. Any initial guess for $(z)$ works well because there is always one minimum, provided that $\left(\alpha_{c}\right)$ and $\left(h_{c}\right)$ remain fixed in the process.

Substituting the depths $\left(z_{c}\right)$ and $\left(h_{c}\right)$ and the dip angle $\left(\alpha_{c}\right)$ in Eq. (1) as fixed parameters, we obtain:

$g\left(x_{i}\right)=K\left[\pi+\operatorname{atan}\left(\frac{x_{i}}{z_{c}}+\cot \alpha_{c}\right)-\operatorname{atan}\left(\frac{x_{i}}{h_{c}}+\cot \alpha_{c}\right)\right]$.

Finally, applying the least-squares method to Eq. (19), the unknown amplitude coefficient $(K)$ can be determined from:

$$
K_{c}=\frac{\sum_{i=1}^{P} L\left(x_{i}\right)\left[\pi+\operatorname{atan}\left(\frac{x_{i}}{z_{c}}+\cot \alpha_{c}\right)-\operatorname{atan}\left(\frac{x_{i}}{h_{c}}+\cot \alpha_{c}\right)\right]}{\sum_{i=1}^{P}\left[\pi+\operatorname{atan}\left(\frac{x_{i}}{z_{c}}+\cot \alpha_{c}\right)-\operatorname{atan}\left(\frac{x_{i}}{h_{c}}+\cot \alpha_{c}\right)\right]^{2}} .
$$


In this way, the problem of determining the depth $(h)$ and the dip angle $(\alpha)$ is transformed into the problem of constructing the depth-dip curves and the problem of depth $(z)$ determination is transformed into the problem of solving a nonlinear least-squares equation, $f(z)=0$, whereas the amplitude coefficient $(K)$ is determined using a simple linear least-squares equation. Eqs. $(13,18,20)$ are ready for determining the model parameters of a buried faulted structure from observed gravity data using a personal computer.

An interpretation scheme based on the above equations for analyzing field data is illustrated in Fig. 2.

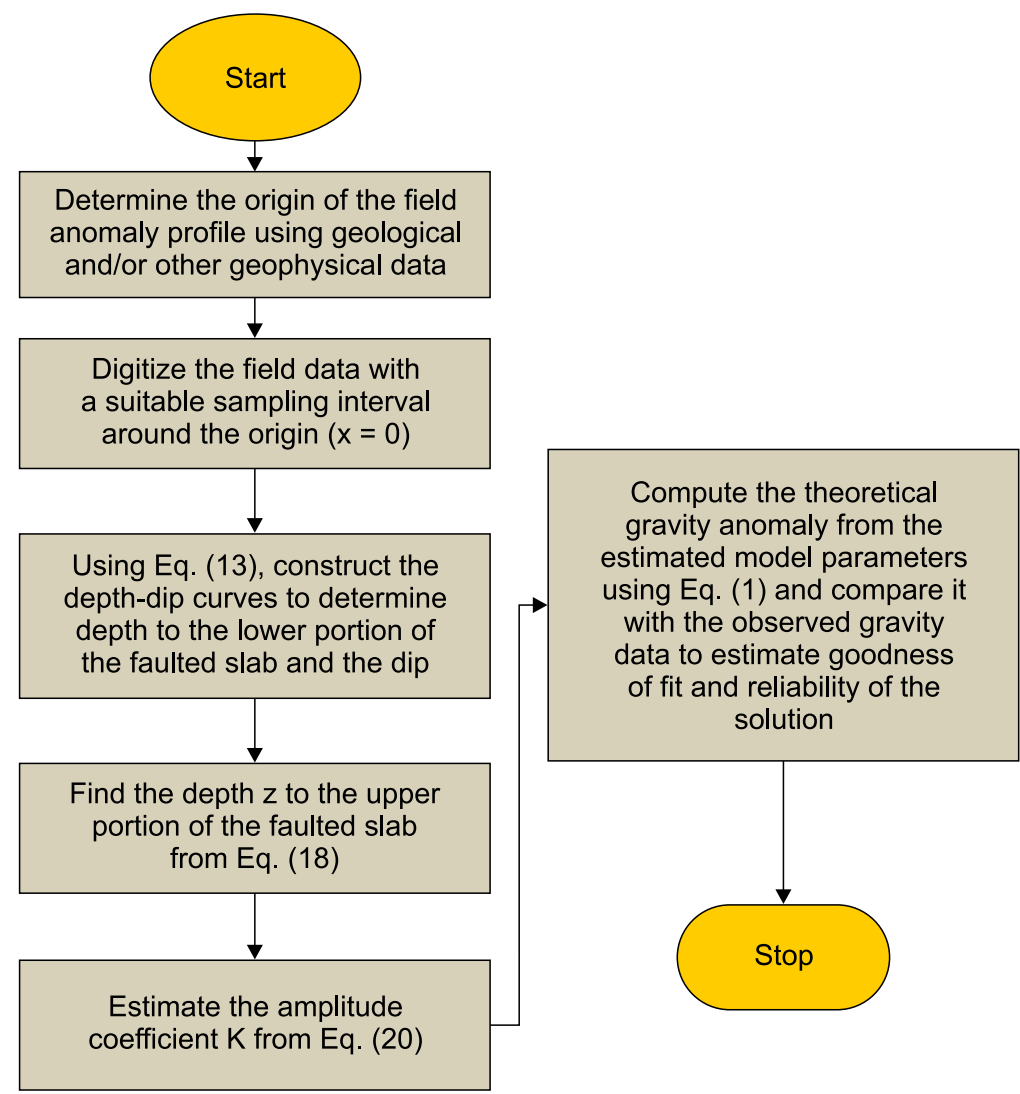

Fig. 2. Generalized scheme for depths, dip angle, and amplitude coefficient determination. 


\section{Theoretical examples:}

\subsection{Error response of the method}

Fig. 3 shows a synthetic gravity anomaly due to a dipping fault $(K=$ $100 \mathrm{mGal}, z=8 \mathrm{~km}, h=12 \mathrm{~km}, \alpha=75^{\circ}$, profile length $=40 \mathrm{~km}$, and sample interval $=1 \mathrm{~km}$ ). The synthetic gravity anomaly was interpreted using our method (Eqs. $(13,18,20))$ to determine, respectively, the depth to the center of the lower portion, the dip angle, the depth to the center of the upper portion, and the amplitude coefficient of the faulted structure. Fig. 4 shows the intersection of the depth-dip curves at the correct location $h=12 \mathrm{~km}$ and $\alpha=75^{\circ}$. The computed depth to the center of the upper portion and the amplitude coefficient determined from Eqs. $(18,20)$ were, respectively, $z=8 \mathrm{~km}$, and $K=100 \mathrm{mGal}$. Perfect results are obtained when using synthetic noise-free data.

Moreover, the synthetic gravity anomaly was contaminated with $5 \%$ random Gaussian error using the following equation:

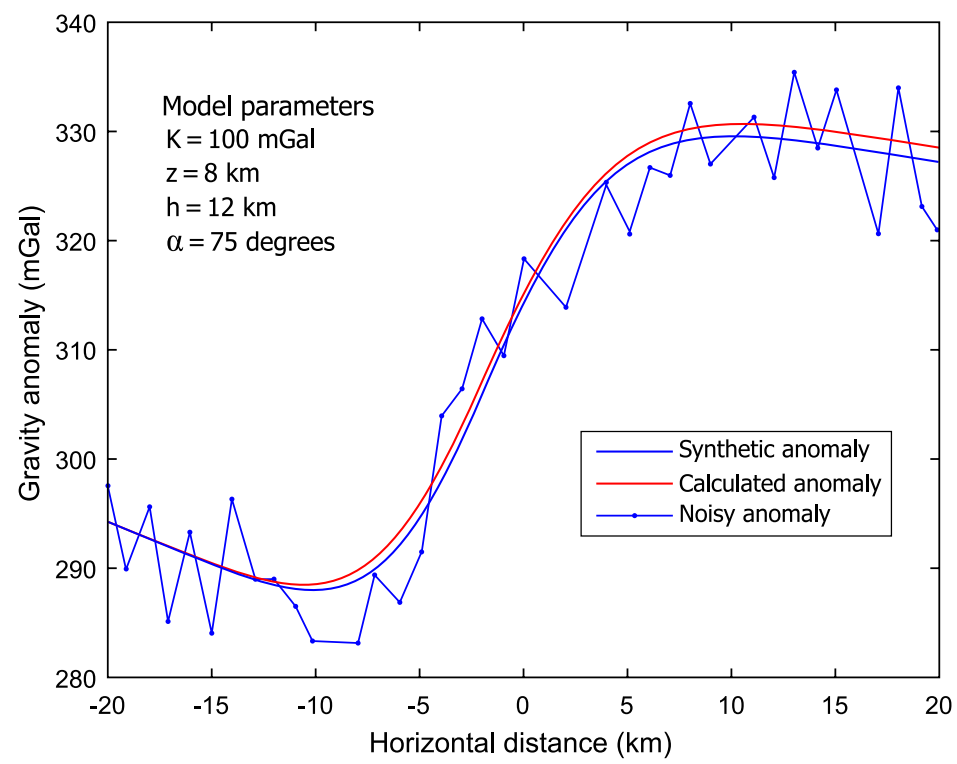

Fig. 3. A synthetic gravity anomaly with and without random errors of a buried dipping fault thin slab. The model parameters are: $K=100 \mathrm{mGal}, z=8 \mathrm{~km}, h=12 \mathrm{~km}$, and $\alpha=75^{\circ}$. The computed gravity anomaly using the estimated model parameters from noisy data is also illustrated. 


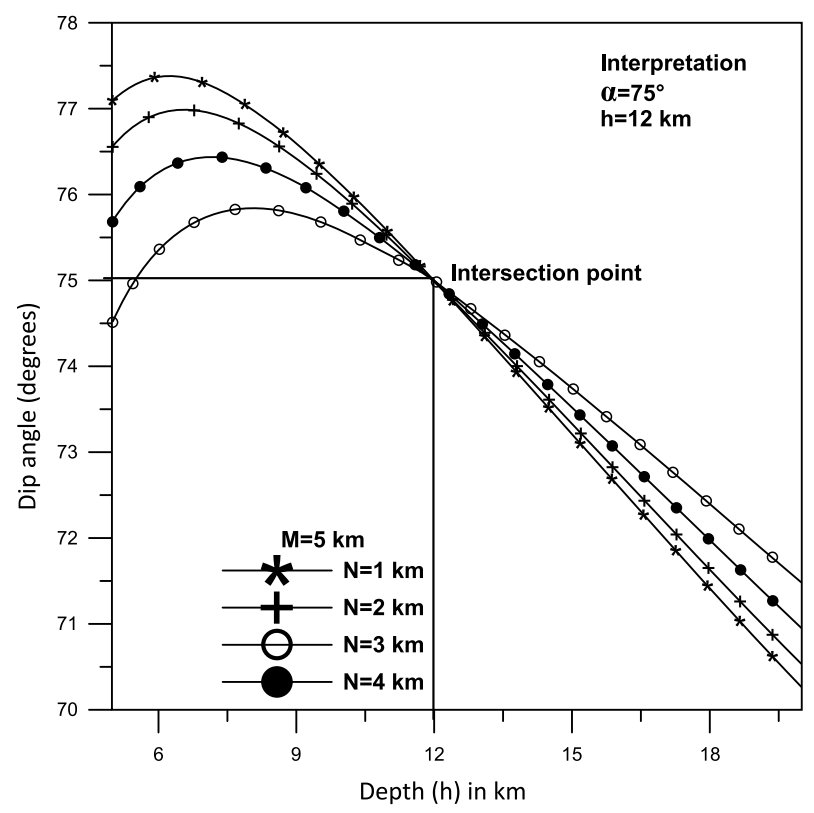

Fig. 4. Interpretation of the data in Fig. 3 using the depth-dip curves method.

$\Delta g_{r n d 1}\left(x_{i}\right)=\Delta g_{1}\left(x_{i}\right)[1+(\operatorname{RND}(i)-0.5) * 0.05]$,

where $\Delta \mathrm{g}_{\text {rnd1 }}\left(x_{i}\right)$ is the contaminated anomaly value at $x_{i}$ and $\operatorname{RND}(i)$ is a pseudo-random number whose range is $(0,1)$. The interval of the pseudo random number is an open interval, i.e. it does not include the extremes values 0 and 1 .

The depth-dip curves method (Eq. 13) was then applied to the noisy gravity anomaly (Fig. 3). The results are plotted in Fig. 5. In this case, the depth-dip curves intersect each other at $h=12.5 \mathrm{~km}$ and $\alpha=74.8^{\circ}$. Using these values, Eqs. $(18,20)$ were then used to estimate the depth to the center of the upper portion and the amplitude coefficient of the fault. The result is: $z=8.3 \mathrm{~km}$ and $K=100.3 \mathrm{mGal}$. The calculated gravity anomaly using the estimated model parameters is shown also in Fig. 3. This figure shows the numerical fitting between the noisy and the calculated synthetic anomaly values. The best fit is considered as a good criterion for accepting the solution in this example and noisy field conditions. The solution for all the model parameters is in good agreement with the actual model parameters. 


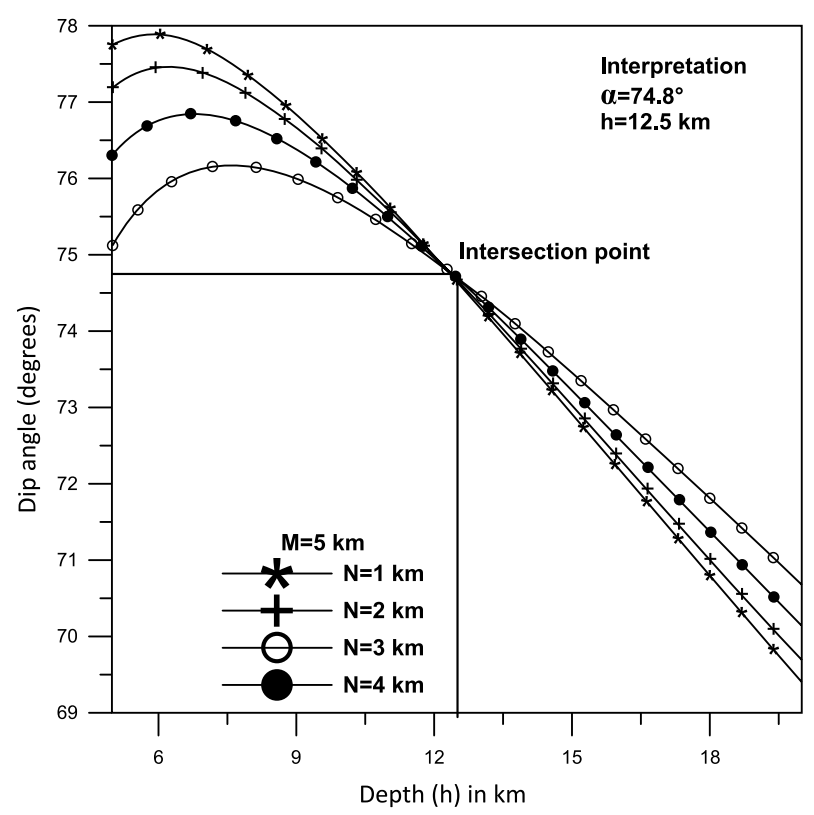

Fig. 5. Interpretation of the data in Fig. 3 after adding 5\% random errors using the present depth-dip curves method.

This demonstrates that our method will give reliable results when applied to noisy gravity data as well.

\subsection{Effect of wrong origin}

Uncertain knowledge of the origin may lead to error in the model parameters $(z, h, \alpha, K)$ when interpreting real data. In this subsection we investigate this effect. The origin of the dipping faulted thin slab was assumed to be chosen incorrectly by introducing errors (offset) of $\pm 500 \mathrm{~m}$ in the horizontal coordinate $x_{i}$ using synthetic data $(K=100 \mathrm{mGal}, z=8 \mathrm{~km}, h=12 \mathrm{~km}$, $\alpha=75^{\circ}$, profile length $=40 \mathrm{~km}$, and sample interval $=1 \mathrm{~km}$ ). Following the same interpretation method, the results are shown in Fig. 6 and Table 1.

It was verified numerically that Eqs. $(13,18,20)$ give an acceptable error of $7.5 \%$ in the model parameters. Fig. 7 shows the gravity anomaly profiles computed using the estimated model parameters (Table 1) compared with the synthetic gravity anomaly. In spite of the error in the calculated model 

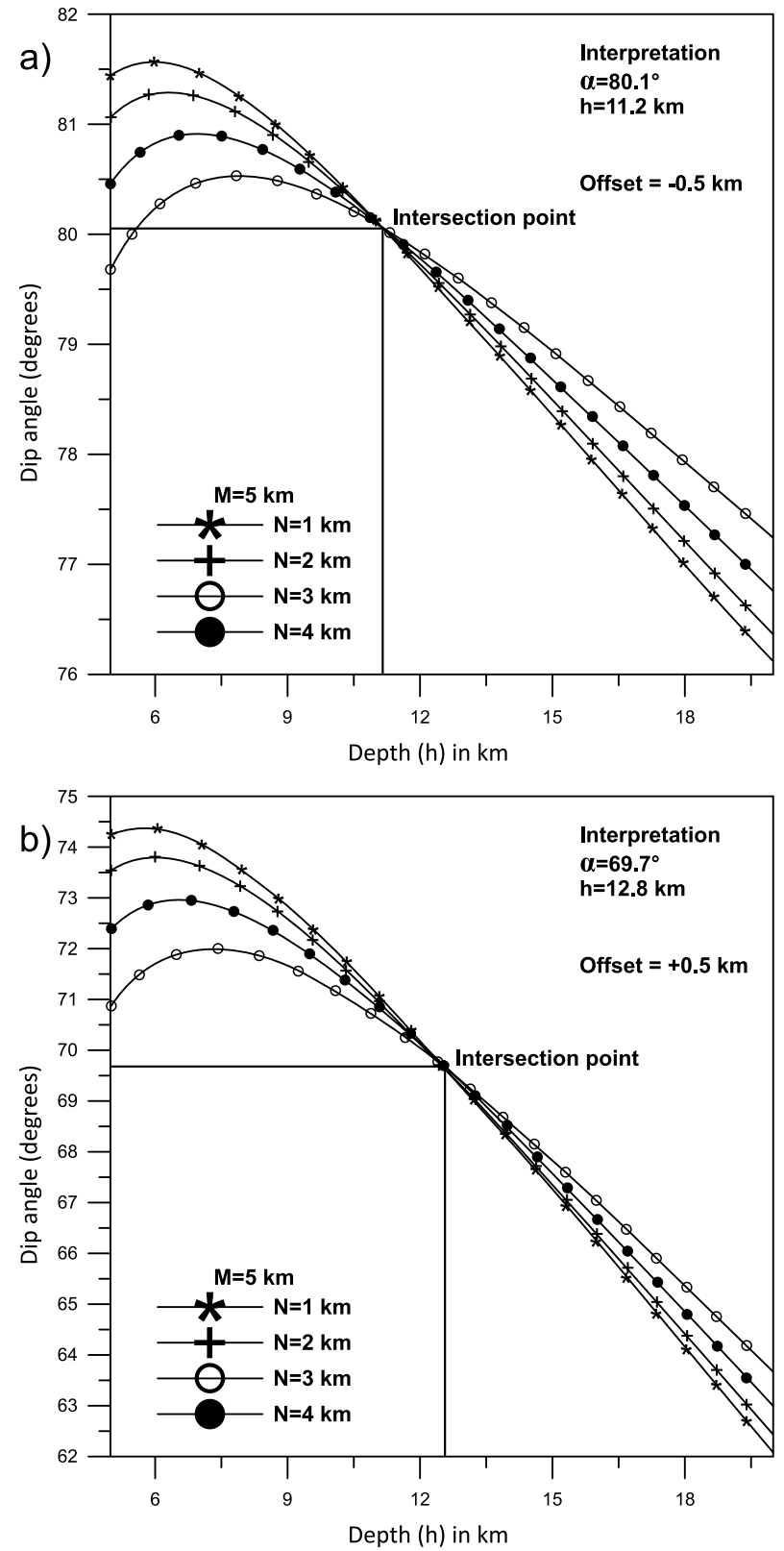

Fig. 6. Data interpretation of Fig. 3 in which offset of $\pm 0.5 \mathrm{~km}$ is introduced into the horizontal position $x_{i}$. 
Table 1. Numerical results obtained from synthetic example $(K=100 \mathrm{mGal}, z=8 \mathrm{~km}$, $h=12 \mathrm{~km}, \alpha=75^{\circ}$, and profile length $\left.=40 \mathrm{~km}\right)$ after adding $\pm 500 \mathrm{~m}$ errors into the horizontal coordinate $x_{i}$.

\begin{tabular}{lcrrr}
\hline Parameters & \multicolumn{2}{c}{ Offset $=-\mathbf{5 0 0} \mathbf{~ m}$} & \multicolumn{2}{c}{ Offset $=\mathbf{+ 5 0 0 ~} \mathbf{m}$} \\
\cline { 2 - 5 } & $\begin{array}{c}\text { Computed } \\
\text { values }\end{array}$ & $\begin{array}{c}\text { \% of } \\
\text { error }\end{array}$ & $\begin{array}{c}\text { Computed } \\
\text { values }\end{array}$ & $\begin{array}{c}\% \text { of } \\
\text { error }\end{array}$ \\
\hline Depth $\boldsymbol{z}(\mathbf{k m})$ & 7.4 & -7.5 & 8.61 & 7.6 \\
\hline Depth $\boldsymbol{h}(\mathbf{k m})$ & 11.2 & -6.7 & 12.8 & 6.7 \\
\hline Dip angle $\boldsymbol{\alpha}($ degrees $)$ & 80.1 & 6.8 & 69.7 & -7.1 \\
\hline Amplitude coefficient $\boldsymbol{K}(\mathbf{m G a l})$ & 99.5 & 0.5 & 100.5 & 0.5 \\
\hline
\end{tabular}

parameters, the overall fitting between the computed and actual gravity anomalies in case the offset is $\pm 500 \mathrm{~m}$ is clear. On the other hand when using larger offset values, the method may result in an instable interpretation curves. However, since the interpretation requires only a relatively short

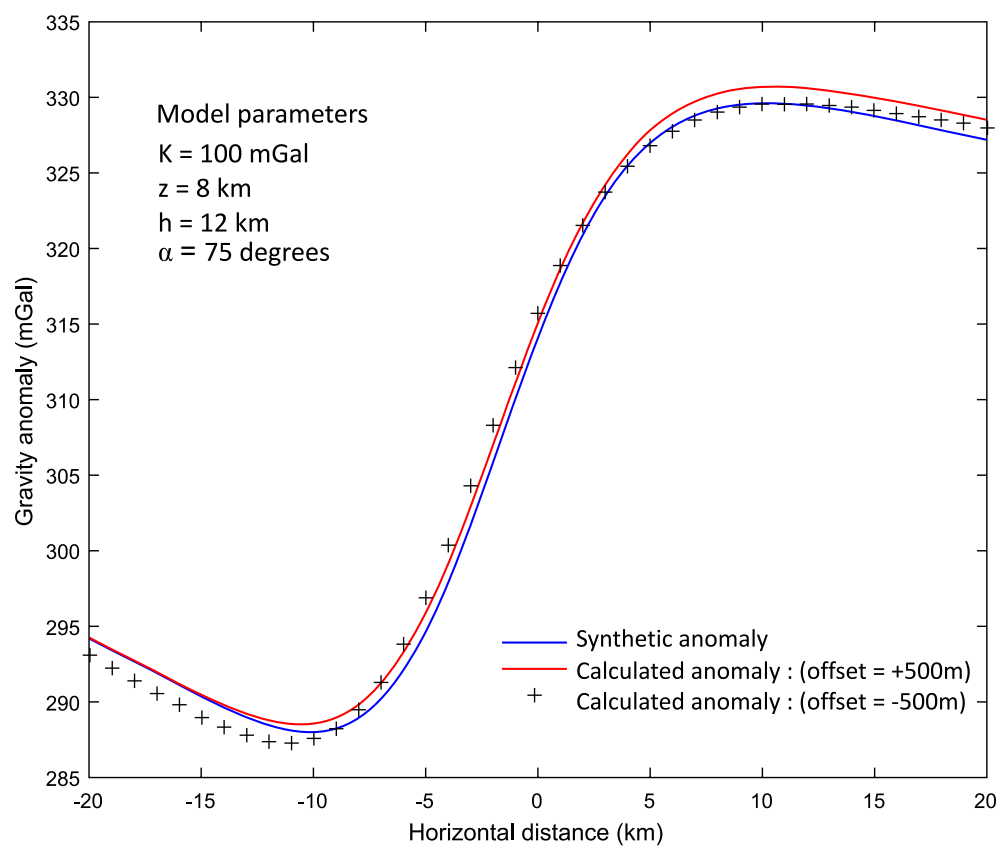

Fig. 7. Synthetic and calculated gravity anomalies using the estimated model parameters after introducing offset of $\pm 500 \mathrm{~m}$. 
profile length, the problem may be solved effectively and economically by increasing the number of measurements made within the restricted length of the profile. At the same time, using a relatively short length of profile, results in a very high rejection of neighboring disturbances. Generally, the accuracy of the result obtained by the present method depends upon the accuracy of which the origin of the fault is determined from geological and/or other geophysical.

\subsection{Effect of using different observation points $N^{\prime} s$ and $M^{\prime} s$}

In the above examples, we have used a fixed $M$ value and different $N$ values to construct the depth-dip curves. In this subsection, we test a large range of $N^{\prime} s$ and $M^{\prime} s$ to investigate whether or not our method would give consistent results.

The synthetic gravity anomaly due to a dipping fault $(K=100 \mathrm{mGal}$, $z=8 \mathrm{~km}, h=12 \mathrm{~km}, \alpha=75^{\circ}$, profile length $=40 \mathrm{~km}$, and sample interval $=1 \mathrm{~km}$ ) shown previously in Fig. 3 was interpreted using the present depthdip curves method using a large range of $N^{\prime} s$ and $M^{\prime} s$. The results are shown in Fig. 8. It is verified that the depth-dip curves intersect at the correct solution $h=12 \mathrm{~km}$ and $\alpha=75^{\circ}$. The depth-dip curves shown in Fig. 8 are similar to the depth-dip curves shown in Fig. 4 but they are not identical because of using different $N$ and $M$ values. This demonstrates that our method will give consistent results when using a large range of $N^{\prime} s$ and $M^{\prime} s$.

\section{Field example}

A Bouguer gravity anomaly profile over the Gazal fault, south Aswan, Egypt is interpreted to determine the model parameters $(z, h, \alpha, K)$. The Bouguer anomaly profile over Gazal fault is shown in Fig. 9. The fault affected both the basement and sedimentary rocks and crops out at the surface (Abdelrahman et al., 1999). The depth to the basement is found to be about $200 \mathrm{~m}$ as obtained from drilling information (Evans et al., 1991). In this example, the fault trace point is determined on the gravity profile, as usual, by projecting the point of intersection between the fault and the 


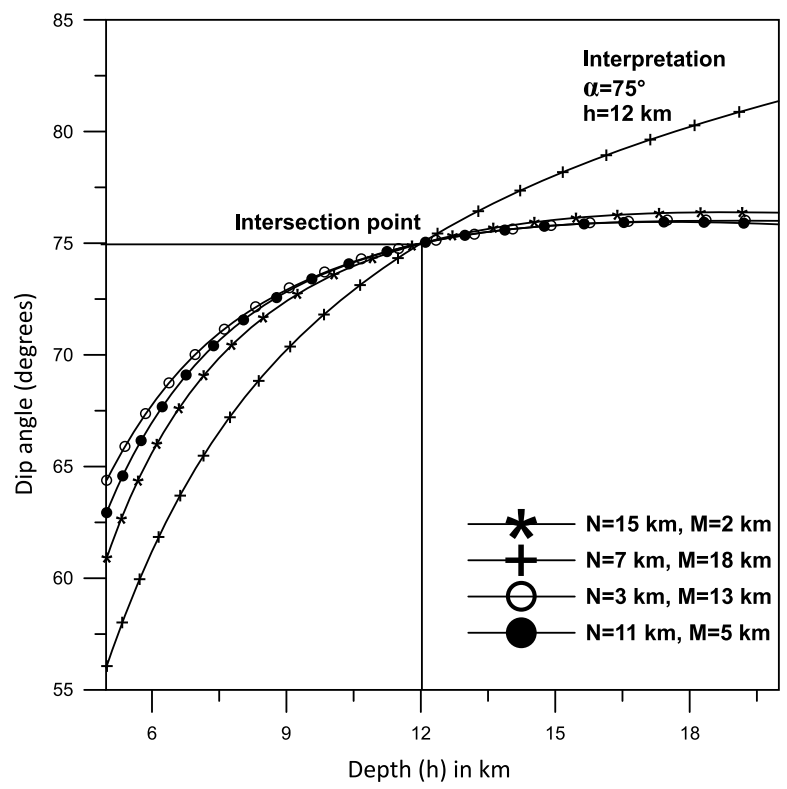

Fig. 8. Interpretation of the data in Fig. 3 using the depth-dip curves method applying a large range of $N^{\prime} s$ and $M^{\prime} s$ values.

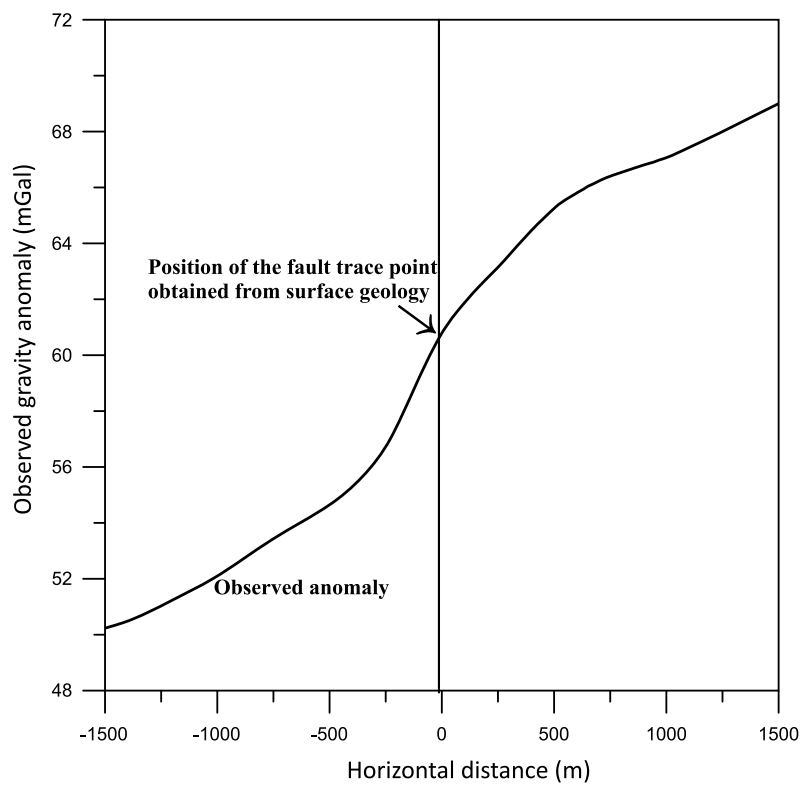

Fig. 9. Gazal Fault gravity anomaly, south Aswan, Egypt. 
ground surface vertically. The gravity profile has been digitized at an interval of $62.5 \mathrm{~m}$. Eq. (13) is applied to the gravity anomaly profile using five combinations of $N$ and $M$ points to construct the depth-dip curves (Fig. 10). This figure shows that the curves can be interpreted to intersect at $h=315 \mathrm{~m}$ and $\alpha=65^{\circ}$. Eqs. $(18,20)$ are used then to determine the depth to the center of the upper portion of the fault slab and the amplitude coefficient. The complete interpretation is: $z=162 \mathrm{~m}, h=315 \mathrm{~m}, \alpha=65^{\circ}$, and $K=19.4 \mathrm{mGal}$. This suggests that Gazal fault resembles a dipping fault $\left(\alpha=65^{\circ}\right)$ where the center of the upper portion of the faulted slab is buried at a depth of $162 \mathrm{~m}$ and the center of the lower portion of the faulted slab is located at a depth of $315 \mathrm{~m}$. The depth to the center to the upper portion of the faulted bed and the dip angle obtained by the present method generally agrees well with those obtained from drilling information and by Abdelrahman et al. (2013), Essa (2013) and Abdelrahman and Essa (2015) as summarized in Table 2. However, the amplitude coefficient obtained by

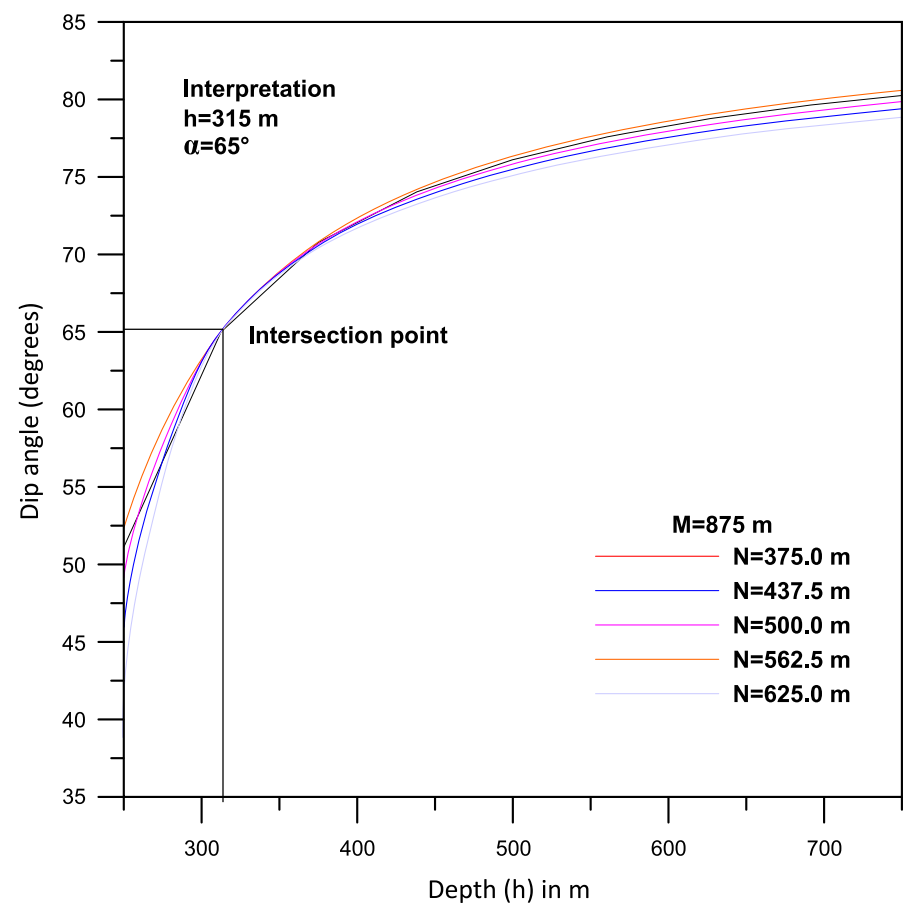

Fig. 10. Data interpretation of Fig. 9 using the depth-dip curves method. 
Table 2. Comparative results of the Gazal field example.

\begin{tabular}{l|cccc}
\hline Parameters & $\begin{array}{c}\text { Abdelrahman } \\
\text { et al. method } \\
(2013)\end{array}$ & $\begin{array}{c}\text { Essa method } \\
(2013)\end{array}$ & $\begin{array}{c}\text { Abdelrahman and } \\
\text { Essa method (2015) }\end{array}$ & $\begin{array}{c}\text { present } \\
\text { method }\end{array}$ \\
\hline Depth $z(\mathrm{~m})$ & 173 & 208 & 202 & 162 \\
Depth $h(\mathrm{~m})$ & - & - & - & 315 \\
Dip angle $\alpha$ (Degrees) & 62.5 & 40 & 58 & 65 \\
\hline Amplitude coefficient & - & - & 2.4 & 19.42 \\
$K$ (mGal) & & & & \\
\hline
\end{tabular}

the present method is extremely larger than that obtained by Abdelrahman and Essa (2015). This is because of the fact that the gravity anomaly over Gazal fault consists of a residual component due to Gazal fault and a regional component represented by a first order polynomial (Abdelrahman and Essa, 2015). It is evident from this field example that our method gives a complete insight from gravity data concerning the nature of Gazal fault structure.

\section{Conclusions}

The problem of determining the depths to the center of the upper and lower portions, dip angle, and amplitude coefficient of a buried dipping faulted thin slab from observed gravity data can be solved using the present method. The present approach is capable of determining completely the model parameters of the buried structure from the gravity data given in a small area over the buried structure, i.e., from the small segment of the gravity profile above the origin. The depths, dip angle, and the amplitude coefficient obtained by present method might be used to gain a complete geologic insight concerning the subsurface. The advantages of the present method over previous graphical and numerical techniques used to interpret gravity data due to dipping faults are: 1) all the four model parameters can be obtained, and 2) the method gives good results when the gravity anomaly is contaminated with Gaussian random noise.

Finally, we envisage the newly introduced method will result in the future development of new methods to interpret completely gravity gradient data due to dipping faults. 
Acknowledgements. The authors would like to thank the editors and the capable anonymous reviewer for their excellent suggestions and thorough review that improved our original manuscript. The authors would like also to thank Prof. Afif Saad (previous Associate Editor of Potential Field Methods, GEOPHYSICS) for reviewing critically and editing the manuscript.

\section{References}

Abdelrahman E. M., Essa K., Abo-Ezz, E. R., 2013: A least-squares window curves method to interpret gravity data due to dipping faults. J. Geophys. Eng., 10, 2, 025003, doi : 10.1088/1742-2132/10/2/025003.

Abdelrahman E. M., Essa K. S., 2015: Three least-squares minimization Approaches to interpret gravity data due to dipping faults. Pure Appl. Geophys., 172, 2, 427-438, doi : $10.1007 / \mathrm{s} 00024-014-0861-4$.

Abdelrahman E. M., Bayoumi A. I., El-Araby H. M., 1989: Dip angle determination of fault planes from gravity data. Pure Appl. Geophys., 130, 4, 735-742, doi : 10.1007 /BF00881608.

Abdelrahman E. M., El-Araby H. M., El-Araby T. M., Abo-Ezz E. R., 2003: A leastsquares derivatives analysis of gravity anomalies due to faulted thin slabs. Geophysics, 68, 2, 535-543, doi : 10.1190/1.1567222.

Abdelrahman E. M., Radwan A. H., Issawy E. A., Al-Araby H. M., Al-Araby T. M., AboEzz E. R. 1999: Gravity interpretation of vertical faults using correlation factors between successive least-squares residual anomalies. The Mining Pribram Symp. on Mathematical Methods in Geology, MC2-1-6.

Chakravarthi V., 2011: Automatic gravity optimization of 2.5D strike listric fault sources with analytically defined fault planes and depth-dependent density. Geophysics, 76 , 2, I21-I31, doi: 10.1190/1.3541957.

Cordell L., Henderson R. G., 1968: Iterative three-dimensional solution of gravity anomaly data using a digital computer. Geophysics, 33, 4, 596-601, doi: 10.1190/1.1439955.

Essa K. S., 2013: Gravity interpretation of dipping faults using the variance analysis method. J. Geophys. Eng., 10, 1, 015003, doi: 10.1088/1742-2132/10/1/015003.

Evans K., Beavan J., Simpson D., 1991: Estimating aquifer parameters from analysis of forced fluctuations in well level: An example from the Nubian Formation near Aswan, Egypt: 1. Hydrogeological background and large-scale permeability estimates. J. Geophys. Res., 96, B7, 12127-12137, doi : 10.1029/91JB00955.

Geldart L. P., Gill D. E., Sharma B., 1966: Gravity anomalies of two dimensional faults. Geophysics, 31, 2, 372-397, doi: 10.1190/1.1439781.

Green R., 1976: Accurate determination of the dip angle of a geological contact using the gravity method. Geophys. Prospect., 24, 2, 265-272, doi: 10.1111/j.1365-2478. 1976. tb00924.x.

Gupta O. P., 1983: A least-squares approach to depth determination from gravity data. Geophysics, 48, 3, 357-360, doi: 10.1190/1.1441473. 
Gupta O. P., Pokhriyal S. K., 1990: New formula for determining the dip angle of a fault from gravity data. SEG Expanded Abstract, 9, 646-649, doi : 10.1190/1.1890290.

Lines L. R., Treitel S., 1984: A review of least-squares inversion and its application to geophysical problems. Geophys. Prospect., 32, 3, 159-186, doi: 10.1111/j.1365-2478 .1984.tb00726.x.

Paul M. K., Datta S., Banerjee B., 1966: Direct interpretation of two dimensional Structural fault from gravity data. Geophysics, 31, 5, 940-948, doi: 10.1190/1.1439825.

Phillips J. D., Hansen R. O., Blakely R. J., 2007: The use of curvature in potential-field interpretation. Exploration Geophysics, 38, 2, 111-119, doi: 10.1071/EG07014.

Press W. H., Teukolsky S. A., Vetterling W. T., Flannery B. P., 2007: Numerical Recipes, The Art of Scientific Computing, Third edition, Cambridge University Press, Cambridge, 1235 p.

Reid A. B., Allsop J. M., Granser H., Millett A. J., Somerton I. W., 1990: Magnetic interpretation in the three dimensions using Euler deconvolution. Geophysics, 55, 1, 80-91, doi: 10.1190/1.1442774.

Tanner J. G., 1967: An automated method of gravity interpretation. Geophys. J. Roy. Astr. Soc., 13, 339-347, 1-3, doi: 10.1111/j.1365-246X.1967.tb02164.x.

Telford W. M., Geldart L. P., Sheriff R. E., Key D. A., 1976: Applied geophysics. Cambridge University Press, London, $860 \mathrm{p}$.

Thompson D. T., 1982: EULDPTH: A new technique for making computer-depth estimates from magnetic data. Geophysics, 47, 1, 31-37, doi : 10.1190/1.1441278.

Utyupin Yu. U., Mishenin S. G., 2012: Locating the sources of potential fields in aerial data using the singularity method. Russ. Geol. Geophys., 53, 10, 1111-1116, doi: $10.1016 / j \cdot r g g \cdot 2012.08 .011$. 